\title{
The asymptpotics of eigenvalues and trace formula of operator associated with one singular problem
}

\author{
Nigar M Aslanova ${ }^{1,2}$
}

\section{Correspondence: nigar. aslanova@yahoo.com \\ ${ }^{1}$ Institute of Mathematics and Mechanics of NAS of Azerbaijan, Baku, Azerbaijan \\ Full list of author information is available at the end of the article}

\section{Abstract}

In the article, spectrum of operator generated by differential operator expression given on semi axis is investigated and proved formula for regularized trace of this operator.

\section{Introduction}

Let $\mathrm{H}$ be a separable Hilbert space with a scalar product (...) and norm $\|$.$\| . Consider in$ $L_{2}((0, \infty), H)$ the problem

$$
\begin{aligned}
& l[y] \equiv-\gamma^{\prime \prime}(x)+x y(x)+A y(x)+q(x) y(x)=\lambda y(x) \\
& y^{\prime}(0)=0,
\end{aligned}
$$

where $A$ is a self-adjoint positive-definite operator in $H$ which has a compact inverse operator and $A>E$ ( $E$ is an identity operator in $H$ ). Denote the eigenvalues and eigenvectors of the operator A by $\gamma_{1} \leq \gamma_{2} \leq \ldots$, and $\phi_{1}, \phi_{2}, \ldots$, respectively.

Suppose that operator-valued function $\mathrm{q}(\mathrm{x})$ is weakly measurable, $\|q(x)\|$ is bounded on $[0, \infty), q^{*}(x)=q(x) \forall x \in[o, \infty)$. The following properties hold:

(1) $\sum_{k=1}^{\infty} \int_{0}^{\infty}\left|\left(q(x) \varphi_{k}, \varphi_{k}\right)\right| d x<$ const, $\forall x \in[0, \infty)$.

(2) $\frac{q_{k}(x)}{x}\left(\left(q(x) \varphi_{k}, \varphi_{k}\right)=q_{k}(x)\right)$ is summable on $(0, \infty), \int_{0}^{\infty} \frac{q_{k}(x)}{x} d x=0$ for $\forall k=\overline{1, \infty}$

(3) $\int_{0}^{\delta} \frac{q_{k}(x)}{x^{5}} d x<\infty, \quad \delta>0, \quad \forall k=\overline{1, \infty}$.

In the case $q(x) \equiv 0$ in $L_{2}(H,(0, \infty))$ associate with problems (1), (2) a self-adjoint operator $L_{0}$ whose domain is

$$
D\left(L_{0}\right)=\left\{\gamma ( x ) \in L _ { 2 } \left(H,(0, \infty) / l[\gamma] \in L_{2}\left(H,(0, \infty), \gamma^{\prime}(0)=0\right\} .\right.\right.
$$

In the case $q(x) \neq 0$ denote the corresponding operator by $L$, so $L=L_{0}+q$.

In this article the asymptotics of eigenvalues and the trace formula of operator $L$ will be studied. 
In [1] the regularized traces of all orders of the operator generated by the expression

$$
l(y) \equiv(-1)^{n} \frac{d^{2 n} y}{d x^{2 n}}+x y
$$

and the boundary conditions

$$
\begin{aligned}
& \sum_{j=0}^{k m} a_{m j} y^{\left(k_{m-j}\right)}(0)=0, \quad m=\overline{1, n} \\
& a_{m 0}=1, k_{n}<k_{n-1}<\cdots<k_{1}<2 n
\end{aligned}
$$

are obtained.

In [2] the sum of eigenvalue differences of two singular Sturm-Liouville operators is studied.

The asymptotics of eigenvalues and trace formulas for operators generated by differential expressions with operator coefficients are studied, for example, in [3-7]. We could also refer to papers [8-10] where trace formulas for abstract operators are obtained. Trace formulas are used for evaluation of first eigenvalues, they have application to inverse problems, index theory of operators and so forth. For further detailed discussions of the subject refer to [11].

\section{The asymptotic formula for eigenvalues of $L_{0}$ and $L$}

One could easily show that under conditions $A>E, A^{-1} \in \sigma_{\infty}$, the spectrum of $L_{0}$ is discrete.

Suppose that $\gamma_{k} \sim a k^{\alpha}(k \rightarrow \infty, a>0, \alpha>0)$. Denote $y_{k}(x)=\left(y(x), \phi_{k}\right)$. Then by virtue of the spectral expansion of the self-adjoint operator $A$ we get the following boundary-value problem for the coefficients $y_{k}(x)$ :

$$
\begin{aligned}
& -y_{k}^{\prime \prime}(x)+x y_{k}(x)+\gamma_{k} y_{k}(x)=\lambda y_{k}(x), \\
& y_{k}^{\prime}(0)=0 .
\end{aligned}
$$

In the case $x+\gamma_{k}>\lambda$ solution of problem (1.1) from $L_{2}(0, \infty)$ is

$$
\psi(x, \lambda)=\sqrt{x+\gamma_{k}-\lambda} K_{\frac{1}{3}}\left\{\frac{2}{3}\left(x+y_{k}-\lambda\right)^{\frac{3}{2}}\right\}
$$

and in the case $x+\gamma_{k}<\lambda$ we can write it as a function of real argument as

$$
\begin{gathered}
\psi(x, y)= \\
=\sqrt{\lambda-\gamma_{k}-x}\left\{J_{\frac{1}{3}}\left(\frac{2}{3}\left(\lambda-\gamma_{k}-x\right)^{\frac{3}{2}}\right)+J_{-\frac{1}{3}}\left(\frac{2}{3}\left(\lambda-\gamma_{k}-x\right)^{\frac{3}{2}}\right)\right\} .
\end{gathered}
$$

For this solution to satisfy (1.2) it is necessary and sufficient to hold

$$
\frac{\pi}{\sqrt{3}}\left(\lambda-\gamma_{k}\right)\left\{J_{\frac{1}{3}}\left(\frac{2}{3}\left(\lambda-\gamma_{k}-x\right)^{\frac{3}{2}}\right)+J_{-\frac{1}{3}}\left(\frac{2}{3}\left(\lambda-\gamma_{k}-x\right)^{\frac{3}{2}}\right)\right\}=0
$$

at least for one $\gamma_{k}\left(\lambda \neq \gamma_{k}\right)$. Therefore, the spectrum of the operator $L_{0}$ consists of those real values of $\lambda \neq \gamma_{k}$ such that at least for one $k$ 


$$
z^{2}\left[J_{\frac{2}{3}}\left(\frac{2}{3} z^{3}\right)-J_{-\frac{2}{3}}\left(\frac{2}{3} z^{3}\right)\right]=0
$$

where $z=\sqrt{\lambda-\gamma_{k}}$.

Prove the following two lemmas which we will need further.

Lemma 1.1. Equation (1.6) has only real roots.

Proof. Suppose that $z=i \alpha, \alpha \in R, \alpha \neq 0$. Then the operator associated with problem

$$
\begin{aligned}
& -y_{k}^{\prime \prime}(x)+x y_{k}(x)=z^{2} y_{k}(x) \\
& y_{k}^{\prime}(0)=0
\end{aligned}
$$

is positive and its eigenvalues are squares of the roots of Equation (1.6). So,

$$
\left(-y^{\prime \prime}{ }_{k}(k), y_{k}(k)\right)+\left(x y_{k}(x), y_{k}(x)\right) \geq 0
$$

But

$$
\left(z^{2} y_{k}(x), y_{k}(x)\right)=-\alpha^{2}\left(y_{k}(x), y_{k}(x)\right)<0
$$

which is contradiction. Then $z$ can be only real, otherwise, the selfadjoint operator corresponding to (1.7), (1.8) will have nonreal eigenvalues, which is impossible. The lemma is proved.

Now, find the asymptotics of the solutions of Equation (1.6). By virtue of the asymptotics for large $|z|[[12]$, p. 975]

$$
J_{v}(z)=\sqrt{\frac{2}{\pi z}} \cos \left(z-\frac{v \pi}{2}-\frac{\pi}{4}\right)\left(1+O\left(\frac{1}{2}\right)\right)
$$

we get

$$
\sin \left(\frac{2}{3} z^{3}-\frac{\pi}{4}\right)\left(1+O\left(\frac{1}{2}\right)\right)=0
$$

Hence

$$
z=\sqrt{\frac{3 \pi m}{2}+\frac{3 \pi}{8}+O\left(\frac{1}{m}\right)}=\left(\frac{3 \pi m}{2}\right)^{\frac{1}{3}}+O\left(\frac{1}{m^{\frac{2}{3}}}\right)
$$

where $m$ is a large integer. Therefore, the statement of the following lemma is true.

Lemma 1.2. For the eigenvalues of $L_{0}$ the following asymptotic is true

$$
\lambda_{m, k}=\gamma_{k}+\alpha_{m^{\prime}}^{2} \quad \alpha_{m}=c m^{\frac{1}{3}}+O\left(\frac{1}{m^{\frac{2}{3}}}\right) \text {. }
$$

For large $|z|$ consider the rectangular contour $l$ with vertices at the points

$$
\pm i B, A_{N} \pm i B, \quad A_{N}=\sqrt[3]{\frac{3 \pi N}{2}+\frac{9 \pi}{8}}
$$

which bypasses the origin along the small semicircle on the right side of the imaginary axis. 
The following lemma is true.

Lemma 1.3. For a sufficiently large integer $N$ the number of the roots of the equation inside l is $N+O(1)$.

Proof. For large $|z|$ we have

$$
\begin{gathered}
z^{2}\left[J_{\frac{2}{3}}\left(\frac{2}{3} z^{3}\right)-J_{-\frac{2}{3}}\left(\frac{2}{3} z^{3}\right)\right]=z^{2} \sqrt{\frac{2}{\pi z^{3}}}\left(\cos \left(\frac{2}{3} z^{3}-\frac{7 \pi}{12}\right)-\right. \\
\left.-\cos \left(\frac{2}{3} z^{3}+\frac{\pi}{12}\right)\right)\left(1+O\left(\frac{1}{z}\right)\right)= \\
=z \sqrt{\frac{2}{\pi z}}\left(\sin \left(\frac{2}{3} z^{3}-\frac{\pi}{4}\right)+O\left(\frac{1}{z}\right)\right) .
\end{gathered}
$$

Denote the function in braces on the right hand side of (1.12) by $F(z)$. Then for large $|z|$ by Rouches' theorem the number of the zeros of $F(z)$ inside the contour equals the number of the zeros $\left(\frac{2}{3} z^{3}-\frac{\pi}{4}\right)$. Therefore, the number of the zeros of function

$$
z^{2}\left[J_{\frac{2}{3}}\left(\frac{2}{3} z^{3}\right)-J_{-\frac{2}{3}}\left(\frac{2}{3} z^{3}\right)\right]
$$

inside $l$ is $N+O(1)$.

Now, by using the above results, derive the asymptotic formula for the eigenvalue distribution of $L_{0}$.

Denote the distribution function of $L_{0}$ by $N(\lambda)$. Then

$$
N(\lambda)=\sum_{\lambda_{m, k}<\lambda} 1
$$

So, $N(\lambda)$ is a number of positive integer pairs $(\mathrm{m}, \mathrm{k})$ for which

$$
\gamma_{k}+\alpha_{m}^{2}<\lambda
$$

By Lemma 1.2 for the great values of $m$

$$
(c-\varepsilon) m^{\frac{2}{3}}<\alpha_{m}^{2}<(c+\varepsilon) m^{\frac{2}{3}} .
$$

From the asymptotics of $\gamma_{k}$ we have

$$
(a-\varepsilon) k^{\alpha}<\gamma_{k}<(a+\varepsilon) k^{\alpha} .
$$

Hence, by virtue of Lemmas 1.1 and 1.3

$$
N^{\prime \prime}(\lambda)+O(1)<N(\lambda)<N^{\prime}(\lambda)+O(1)
$$

where $N^{\prime \prime}(\lambda)$ is the number of the positive integer pairs for which

$$
(a+\varepsilon) k^{\alpha}+(c+\varepsilon) m^{\frac{2}{3}}<\lambda,
$$

$N^{\prime}(\lambda)$ is the number of the positive integer pairs $(m, k)$ satisfying the inequality

$$
(c-\varepsilon) m^{\frac{2}{3}}+(a-\varepsilon) k^{\alpha}<\lambda .
$$

Thus by using (1.14), (1.15) in (1.13) as in [[13], Lemma 2] we come to the following statement. 
Lemma 1.4. If $\gamma_{k} \sim a k^{\alpha},(0<a, \alpha>0)$ then

$$
\lambda_{n} \sim \mu_{n} \sim d n^{\delta}
$$

where

$$
\delta= \begin{cases}\frac{2 \alpha}{2+3 \alpha}, & \alpha \in\left(0, \frac{2}{3}\right) \\ \frac{\alpha}{2}, & \alpha>\frac{2}{3} \\ \frac{1}{3}, & \alpha=\frac{2}{3}\end{cases}
$$

\section{Trace formula}

The following lemma is true.

Lemma 2.1. Let the conditions of Lemma 1.4 hold. Then for $\alpha>\frac{2}{3}$ there exists such a subsequence $\left\{n_{m}\right\}$ of natural numbers that the relation

$$
\mu_{k}-\mu_{n_{m}} \geq \frac{d}{2}\left(k^{\frac{\alpha}{2}}-n_{m}^{\frac{\alpha}{2}}\right), \quad k=n_{m}, n_{m}+1, \ldots
$$

holds.

Proof. In virtue of Lemma 1.4 for $\alpha>\frac{2}{3}, \lim _{n \rightarrow \infty} \frac{\mu_{n}}{n^{\frac{\alpha}{2}}}=d$, from which it follows that

$$
\lim _{n \rightarrow \infty}\left(\mu_{n}-\frac{d}{2} n^{\frac{\alpha}{2}}\right)=\infty
$$

That is why one could choose a subsequence $n_{1}<n_{2}<\ldots . n_{m}<\ldots$, that for each $k \geq$ $n_{m}$ holds $\mu_{k}-\frac{d}{2} k^{\frac{\alpha}{2}} \geq \mu_{n_{m}}-\frac{d}{2} n_{m}^{\frac{\alpha}{2}}$, or $\mu_{k}-\mu_{n_{m}} \geq \frac{d}{2}\left(k^{\frac{\alpha}{2}}-n_{m}^{\frac{\alpha}{2}}\right)$. The lemma is proved.

We will call $\lim _{m \rightarrow \infty} \sum_{n=1}^{n_{m}}\left(\lambda_{n}-\mu_{n}\right)$ a regularized trace of the operator $L$. It will be shown later it is independent of the choice of $\left\{n_{m}\right\}$ satisfying the hypothesis of Lemma 2.1.

From (1.16) it is obvious that for $\alpha>2$ resolvents $R\left(L_{0}\right)$ and $R(L)$ are trace class operators. By using Lemma 2.1 for $\alpha>2$ one can prove the following lemma.

Lemma 2.2. Let $\|q(x)\|<$ const on the interval $[0, \infty)$ and also the conditions of Lemma 1.6 hold. Then for $\alpha>2$

$$
\lim _{m \rightarrow \infty} \sum_{n=1}^{n_{m}}\left(\lambda_{n}-\mu_{n}-\left(q \psi_{n}, \psi_{n}\right)\right)=0,
$$

where $\left\{\psi_{n}\right\}$ are orthonormal eigenvectors of the operator $L_{0}$.

The proof of this lemma is analogous to the proof of Lemma 2 and Theorem 2 from [8]. For this reason we will not derive it here.

The orthogonal eigen-vectors of the operator $L_{0}$ in $L_{2}((0, \infty), H)$ are

$$
\psi_{m, k}=c_{m, k} \psi\left(x, \alpha_{m}^{2}\right) \varphi_{k}
$$


Calculate their norm. We have

$$
\left\|\psi_{m, k}\right\|^{2}=c_{m, k}^{2} \int_{0}^{\infty} \psi\left(x, \alpha_{m}^{2}\right)^{2} d x .
$$

Take in Equation (1.7) $z^{2}=\alpha^{2}$ and $z^{2}=\beta^{2}$. The solutions corresponding to these values denote by $\psi\left(x, \alpha^{2}\right)$ and $\psi\left(x, \beta^{2}\right)$. Multiplying the first of the obtained equations by $\psi\left(x, \beta^{2}\right)$, the second by $\psi\left(x, \alpha^{2}\right)$, subtracting the second one from the first one and integrating from zero to infinity we get

$$
\begin{gathered}
\int_{0}^{\infty} \psi\left(x, \alpha^{2}\right) \psi\left(x, \beta^{2}\right) d x=\frac{\psi\left(0, \alpha^{2}\right)^{\prime} \psi\left(0, \beta^{2}\right)-\psi\left(0, \alpha^{2}\right) \psi\left(0, \beta^{2}\right)^{\prime}}{\alpha^{2}-\beta^{2}}= \\
\frac{\pi^{2}}{3} \alpha \beta\left[\frac{\alpha\left\{J_{\frac{2}{3}}\left(\frac{2}{3} \alpha^{3}\right)-J_{-\frac{2}{3}}\left(\frac{2}{3} \alpha^{3}\right)\right\}\left\{J_{\frac{1}{3}}\left(\frac{2}{3} \beta^{3}\right)+J_{-\frac{1}{3}}\left(\frac{2}{3} \beta^{3}\right)\right\}}{\alpha^{2}-\beta^{2}}\right. \\
\left.\quad \frac{\beta\left\{J_{\frac{1}{3}}\left(\frac{2}{3} \alpha^{3}\right)+J_{-\frac{1}{3}}\left(\frac{2}{3} \alpha^{3}\right)\right\}\left\{J_{\frac{2}{3}}\left(\frac{2}{3} \beta^{3}\right)-J_{-\frac{2}{3}}\left(\frac{2}{3} \beta^{3}\right)\right\}}{\alpha^{2}-\beta^{2}}\right] .
\end{gathered}
$$

Going to limit as $\alpha \rightarrow \beta$, we get

$$
\begin{gathered}
\int_{0}^{\infty} \psi\left(x, \alpha^{2}\right)^{2} d x= \\
=\frac{\pi^{2}}{6}\left[\beta\left(J_{\frac{2}{3}}\left(\frac{2}{3} \beta^{3}\right)-J_{-\frac{2}{3}}\left(\frac{2}{3} \beta^{3}\right)\right)\left(J_{\frac{1}{3}}\left(\frac{2}{3} \beta^{3}\right)+J_{-\frac{1}{3}}\left(\frac{2}{3} \beta^{3}\right)\right)+\right. \\
+\beta^{2}\left\{J_{\frac{1}{3}}\left(\frac{2}{3} \beta^{3}\right)+J_{-\frac{1}{3}}\left(\frac{2}{3} \beta^{3}\right)\right)\left(J_{\frac{2}{3}}\left(\frac{2}{3} \alpha^{3}\right)-J_{-\frac{2}{3}}\left(\frac{2}{3} \alpha^{3}\right)\right)_{\alpha=\beta}^{\prime}- \\
-\left(J_{\frac{1}{3}}\left(\frac{2}{3} \alpha^{3}\right)+J_{-\frac{1}{3}}\left(\frac{2}{3} \alpha^{3}\right)\right)_{\alpha=\beta}^{\prime}\left(J_{\frac{2}{3}}\left(\frac{2}{3} \beta^{3}\right)-J_{-\frac{2}{3}}\left(\frac{2}{3} \beta^{3}\right)\right) .
\end{gathered}
$$

By making use of identities (12, p.981)

$$
\begin{aligned}
& z J_{v}^{\prime}(z)+v J_{v}(z)=z J_{v-1}(z) \\
& z J_{v}^{\prime}(z)-v J_{v}(z)=-z J_{v+1}(z),
\end{aligned}
$$

we have

$$
\begin{gathered}
\int_{0}^{\infty} \psi\left(x, \alpha^{2}\right)^{2} d x=\frac{\pi^{2}}{3} \alpha^{4} \times \\
\times\left[\left(J_{\frac{1}{3}}\left(\frac{2}{3} \alpha^{3}\right)+J_{-\frac{1}{3}}\left(\frac{2}{3} \alpha^{3}\right)\right)^{2}+\left(J_{\frac{1}{3}}\left(\frac{2}{3} \alpha^{3}\right)+J_{-\frac{1}{3}}\left(\frac{2}{3} \alpha^{3}\right)\right)^{2}\right] .
\end{gathered}
$$


Finally by equation

$$
\beta^{2}\left\{J_{\frac{2}{3}}\left(\frac{2}{3} \beta^{3}\right)-J_{-\frac{2}{3}}\left(\frac{2}{3} \beta^{3}\right)\right\}=0
$$

we get

$$
\left\|\psi_{m, k}\right\|^{2}=c_{m, k}^{2} \frac{\pi^{2}}{3} \alpha_{m}^{4}\left(J_{\frac{1}{3}}\left(\frac{2}{3} \alpha^{3}\right)+J_{-\frac{1}{3}}\left(\frac{2}{3} \alpha^{3}\right)\right)^{2} .
$$

So, the orthonormal eigenvectors of $L_{0}$ are

$$
\psi_{m, k}=\frac{\sqrt{3} \psi\left(x, \alpha_{m}^{2}\right)}{\pi \alpha_{m}^{2}\left(J_{\frac{1}{3}}\left(\frac{2}{3} \alpha_{m}^{3}\right)+J_{-\frac{1}{3}}\left(\frac{2}{3} \alpha_{m}^{3}\right)\right)} \varphi_{k}
$$

Lemma 2.3. If the operator-valued function $q(x)$ has property 1 and $\alpha>\frac{2}{3}$, then

$$
\frac{3}{\pi^{2}} \sum_{k=1}^{\infty} \sum_{m=1}^{\infty}\left|\int_{0}^{\infty} \frac{\left(q(x) \varphi_{k}, \varphi_{k}\right) \psi\left(x, \alpha_{m}^{2}\right)^{2} d x}{\alpha_{m}^{4}\left(J_{\frac{1}{3}}\left(\frac{2}{3} \alpha_{m}^{3}\right)+J_{-\frac{1}{3}}\left(\frac{2}{3} \alpha_{m}^{3}\right)\right)^{2}}\right|<\infty
$$

Proof. Take $\left(q(x) \phi_{k}, \phi_{k}\right)=q_{k}(x)$. Let $\varepsilon>0$ be sufficiently small number. If $x \in\left(0, \alpha_{m}^{2}-\alpha_{m}^{\varepsilon}\right)$ then $z=\alpha_{m}^{2}-x \in\left(\alpha_{m}^{2}, \alpha_{m}^{\varepsilon}\right)$. For $x \in\left(\alpha_{m}^{2}-\alpha_{m}^{\varepsilon}, \alpha_{m}^{2}+\alpha_{m}^{\varepsilon}\right)$ we have $z \in\left(-\alpha_{m}^{\varepsilon}, 0\right] \cup\left(0, \alpha_{m}^{\varepsilon}\right)$ and, finally, for $x \in\left(\alpha_{m}^{2}+\alpha_{m}^{\varepsilon}+\infty\right)$ it will be $z \in\left(-\infty,-\alpha_{m}^{\varepsilon}\right)$.

Consequently for $z \in\left(\alpha_{m}^{2}, \alpha_{m}^{\varepsilon}\right)$ we have

$$
\psi\left(x, \alpha_{m}^{2}\right)=\sqrt{\alpha_{m}^{2}-x}\left(J_{\frac{1}{3}}\left(\frac{2}{3} \alpha_{m}^{2}-x\right)^{\frac{3}{2}}+J_{-\frac{1}{3}}\left(\frac{2}{3} \alpha_{m}^{2}-x\right)^{\frac{3}{2}}\right) \sim \frac{e^{-i \sqrt{z} 3}}{z}
$$

and for $z \in\left(-\infty,-\alpha_{m}^{3}\right)$

$$
\psi\left(x, \alpha_{m}^{2}\right)=\sqrt{x-\alpha_{m}^{2}} K_{\frac{1}{3}}\left(\frac{2}{3}\left(x-\alpha_{m}^{2}\right)^{\frac{3}{2}}\right) \sim \frac{e^{-\sqrt{-z}^{3}}}{-z}
$$

then

$$
\begin{aligned}
& \left|\int_{0}^{\infty} q_{k}(x) \psi\left(x, \alpha_{m}^{2}\right) d x\right| \sim \mid \int_{\alpha_{m}^{2}}^{\alpha_{m}^{\varepsilon}} \frac{e^{-2 i \sqrt{-z}}}{z^{2}} q_{k}\left(\alpha_{m}^{2}-z\right) d z+ \\
& +\int_{\alpha_{m}^{2}}^{\alpha_{m}^{\varepsilon}} q_{k}\left(\alpha_{m}^{2}-z\right) \psi^{2}(z) d z+\int_{-\infty}^{-\alpha_{m}^{\varepsilon}} \frac{e^{-2 \sqrt{-z^{3}}}}{z^{2}} q_{k}\left(\alpha_{m}^{2}-z\right) d z \mid< \\
< & \int_{0}^{\infty}\left|q_{k}(z)\right| d z+\int_{\alpha_{m}^{\varepsilon}}^{-\alpha_{m}^{\varepsilon}}\left|q_{k}\left(\alpha_{m}^{2}-z\right) \psi^{2}(z)\right| d z+\int_{0}^{\infty}\left|q_{k}(z) d z\right| .
\end{aligned}
$$


For $\varepsilon \rightarrow 0$ we have

$$
\begin{gathered}
\lim _{\varepsilon \rightarrow 0} \int_{\alpha_{m}^{\varepsilon}}^{-\alpha_{m}^{\varepsilon}}\left|q_{k}\left(\alpha_{m}^{2}-z\right) \psi^{2}(z)\right| d z= \\
=\int_{-1}^{1}\left|q_{k}\left(a_{m}^{2}-z\right) \psi^{2}(z)\right| d z<c \int_{-1}^{1}\left|q_{k}(z)\right| d z<\infty .
\end{gathered}
$$

From asymptotic $\alpha_{m} \sim \mathrm{cm}^{\frac{1}{3}}$ by using (2.10), (2.11) and property 1 we get

$$
\begin{gathered}
\sum_{k=1}^{\infty} \sum_{m=1}^{\infty} \frac{3}{\pi^{2}} \int_{0}^{\infty}\left|\frac{q_{k}(x) \psi\left(x, \alpha_{m}^{2}\right)^{2} d x}{\alpha_{m}^{4}\left(J_{\frac{1}{3}}\left(\frac{2}{3} \alpha_{m}^{3}\right)+J_{-\frac{1}{3}}\left(\frac{2}{3} \alpha_{m}^{3}\right)\right)^{2}}\right|< \\
<\sum_{k=1}^{\infty} \int_{0}^{\infty}\left|q_{k}(x)\right| d x \sum_{m=1}^{\infty} \frac{1}{m^{\frac{4}{3}}}<\infty
\end{gathered}
$$

The lemma is proved.

By using Lemma 2.3 prove the following theorem.

Theorem 2.1. Let the conditions of Lemma 1.6 hold. If the operator-valued function $q$ (x) has properties 1-3, then it holds the formula

$$
\lim _{m \rightarrow \infty} \sum_{n=1}^{n_{m}}\left(\lambda_{n}-\mu_{n}\right)=0 .
$$

Proof. In virtue of Lemma 2.1

$$
\lim _{m \rightarrow \infty} \sum_{n=1}^{n_{m}}\left(\lambda_{n}-\mu_{n}\right)=\sum_{k=1}^{\infty} \sum_{m=1}^{\infty} \int_{0}^{\infty} \frac{3}{\pi} \frac{q_{k}(x) \psi\left(x, \alpha_{m}^{2}\right)^{2} d x}{\alpha_{m}^{4}\left(J_{\frac{1}{3}}\left(\frac{2}{3} \alpha_{m}^{3}\right)+J_{-\frac{1}{3}}\left(\frac{2}{3} \alpha_{m}^{3}\right)\right)^{2}}
$$

Denote

$$
T_{N}(x)=\sum_{m=1}^{N} \frac{3}{\pi^{2}} \frac{\psi\left(x, \alpha_{m}^{2}\right)^{2}}{\alpha_{m}^{4}\left(J_{\frac{1}{3}}\left(\frac{2}{3} \alpha_{m}^{3}\right)+J_{-\frac{1}{3}}\left(\frac{2}{3} \alpha_{m}^{3}\right)\right)} .
$$

Show that for each fixed value of $k$ the $m$-th term of the sum $T_{N}(x)$ is a residue at the point $\alpha_{m}$ of some function of complex variable which has poles at points $\alpha_{m}(m=\overline{1, N})$.

For this purpose consider the following function

$$
\begin{gathered}
g(z)=\frac{\pi^{2}}{3}\left(z^{2}-x\right)^{2} \frac{\left(J_{\frac{1}{3}}\left(\frac{2}{3}\left(z^{2}-x\right)^{\frac{3}{2}}\right)+J_{-\frac{1}{3}}\left(\frac{2}{3}\left(z^{2}-x\right)^{\frac{2}{3}}\right)\right)^{2}}{2 z\left(J_{\frac{2}{3}}\left(\frac{2}{3} z^{3}\right)-J_{-\frac{2}{3}}\left(\frac{2}{3} z^{3}\right)\right)^{2}}+ \\
+\frac{J_{\frac{2}{3}}\left(\left(\frac{2}{3}\left(z^{2}-x\right)^{\frac{3}{2}}\right)-J_{-\frac{2}{3}}\left(\frac{2}{3}\left(z^{2}-x\right)^{\frac{3}{2}}\right)\right)^{2}}{2 z\left(J_{\frac{2}{3}}\left(\frac{2}{3} z^{3}\right)-J_{-\frac{2}{3}}\left(\frac{2}{3} z^{3}\right)\right)^{2}} .
\end{gathered}
$$


By taking in place of zero $x$ in (2.6) one can show that

$$
\begin{gathered}
\int_{x}^{\infty} \psi\left(t, z^{2}\right)^{2} d t=\frac{\pi^{2}}{3}\left(z^{2}-x\right)^{2}\left[\left(J_{\frac{1}{3}}\left(\frac{2}{3}\left(z^{2}-x\right)^{\frac{3}{2}}\right)+J_{-\frac{1}{3}}\left(\frac{2}{3}\left(z^{2}-x\right)^{\frac{3}{2}}\right)\right)^{2}+\right. \\
\left.+\left(J_{\frac{2}{3}}\left(\frac{2}{3}\left(z^{2}-x\right)^{\frac{3}{2}}\right)-J_{-\frac{2}{3}}\left(\frac{2}{3}\left(z^{2}-x\right)^{\frac{3}{2}}\right)\right)^{2}\right] .
\end{gathered}
$$

Note that all zeros of the function $J_{\frac{2}{3}}\left(\frac{2}{3} z^{3}\right)-J_{-\frac{2}{3}}\left(\frac{2}{3} z^{3}\right)$ are simple, otherwise

$$
\begin{gathered}
\left(J_{\frac{2}{3}}\left(\frac{2}{3} z^{3}\right)-J_{-\frac{2}{3}}\left(\frac{2}{3} z^{3}\right)\right)_{z=\alpha m}^{\prime}= \\
=2 \alpha_{m}^{2}\left(J_{-\frac{1}{3}}\left(\frac{2}{3} \alpha_{m}^{3}\right)+J_{\frac{1}{3}}\left(\frac{2}{3} \alpha_{m}^{3}\right)-\frac{1}{\alpha_{m}^{3}}\left(J_{\frac{2}{3}}\left(\frac{2}{3} \alpha_{m}^{3}\right)-J_{-\frac{2}{3}}\left(\frac{2}{3} \alpha_{m}^{3}\right)\right)\right)= \\
=2 \alpha_{m}^{2}\left(J_{-\frac{1}{3}}\left(\frac{2}{3} \alpha_{m}^{3}\right)+J_{\frac{1}{3}}\left(\frac{2}{3} \alpha_{m}^{3}\right)\right)=0
\end{gathered}
$$

and by virtue of (2.7) the norm of the eigenvectors equals zero, which is contradiction.

Denote $z^{2}-x=f(x, z)$ and the right hand side of (2.14) by $G(f(x, z)$. Then

$$
G_{x}^{\prime}=-G_{f}^{\prime}, G_{z}^{\prime}=2 z G_{f}^{\prime}=-2 z G_{x}^{\prime} .
$$

Then from (2.14), (2.15)

$$
G_{x}^{\prime}=\psi\left(x, z^{2}\right)^{2}, G_{z}^{\prime}=2 z \psi\left(x, z^{2}\right)^{2} .
$$

The function $g(z)$ has poles of second order at the points $\alpha_{m}$. By using identities (2.15), (2.16) show that residues at this points equal the terms of sum $T_{N}(x)$. Denoting $J_{\frac{2}{3}}\left(\frac{2}{3} z^{3}\right)-J_{-\frac{2}{3}}\left(\frac{2}{3} z^{3}\right)=u(z)$, write Taylor expansion of this function in the vicinity $\alpha_{m}:$

$$
\begin{gathered}
u(z)=\left(z-\alpha_{m}\right) u^{\prime}\left(\alpha_{m}\right)+\frac{\left(z-\alpha_{m}\right)^{2}}{2 !} u^{\prime \prime}\left(\alpha_{m}\right)+\cdots \\
u^{2}(z)=\left(z-\alpha_{m}\right)^{2} u^{\prime}\left(\alpha_{m}\right)^{2}+\left(z-\alpha_{m}\right)^{3} u^{\prime}\left(\alpha_{m}\right) u^{\prime \prime}\left(\alpha_{m}\right)+\cdots
\end{gathered}
$$

Show that the coefficient of the expansion of function $z u^{2}(z)$ at $\left(z-\alpha_{m}\right)^{3}$ equals zero. So,

$$
\begin{gathered}
z u^{2}(z)=\left(\left(z-\alpha_{m}\right)+\alpha_{m}\right) u^{2}(z)=\alpha_{m} u^{\prime}\left(\alpha_{m}\right)^{2}\left(z-\alpha_{m}\right)^{2}+ \\
+u^{\prime}\left(\alpha_{m}\right)\left(\alpha_{m} u^{\prime \prime}\left(\alpha_{m}\right)+u^{\prime}\left(\alpha_{m}\right)\right)\left(z-\alpha_{m}\right)^{3}+\cdots
\end{gathered}
$$

By denoting $\frac{2}{3} z^{3}=w(z)$ we have

$$
u^{\prime}\left(\alpha_{m}\right)=2 \alpha_{m}^{2}\left(J_{\frac{2}{3}}(w(z))-J_{-\frac{2}{3}}(w(z))\right)_{w=\frac{2}{3} \alpha_{m}^{3}}^{\prime}
$$




$$
\begin{gathered}
u^{\prime \prime}\left(\alpha_{m}\right)=4 \alpha_{m}^{4}\left(J_{\frac{2}{3}}(w(z))-J_{-\frac{2}{3}}(w(z))\right)^{\prime \prime}+ \\
+4 \alpha_{m}\left(J_{\frac{2}{3}}(w(z))-J_{-\frac{2}{3}}(w(z))\right)_{w=\frac{2}{3} \alpha_{m}^{3}} .
\end{gathered}
$$

Therefore,

$$
\alpha_{m} u^{\prime \prime}\left(\alpha_{m}\right)+u^{\prime}\left(\alpha_{m}\right)=2 \alpha_{m}^{2}\left(2 \alpha_{m}^{3} u_{w}^{\prime \prime}+3 u_{w}^{\prime}\right)_{w=\frac{2}{3} \alpha_{m}^{3}}
$$

On the other hand, $J_{\frac{2}{3}}(z)$ and $J_{-\frac{2}{3}}(z)$ satisfy the Bessel equation $z^{2} \frac{d^{2} y}{d z^{2}}+z \frac{d y}{d z}+\left(z^{2}-v^{2}\right) y=0$ for $v^{2}=\frac{4}{9}$. So their difference also satisfies this equation

$$
u_{w}^{\prime \prime} w^{2}+w u_{w}^{\prime}=\left(v^{2}-w^{2}\right) u
$$

If $w=\frac{2}{3} \alpha_{m}^{3}$, then the right hand side (2.21) vanishes. Hence,

$$
u_{w}^{\prime \prime} w^{2}+w u_{w}^{\prime}=\frac{2}{9} \alpha_{m}^{3}\left[2 \alpha_{m}^{3} u_{w=\frac{2}{3} \alpha_{m}^{3}}+3 u_{w=\frac{2}{3} \alpha_{m}^{3}}^{\prime}\right]=0
$$

which shows that the coefficient at $\left(z-\alpha_{m}\right)^{3}$ in (2.17) vanishes.

Consequently, by (2.16), (2.17), (2.22) and the relation

$$
\begin{gathered}
\left(J_{\frac{2}{3}}\left(\frac{2}{3} z^{3}\right)-J_{-\frac{2}{3}}\left(\frac{2}{3} z^{3}\right)\right)_{z=\alpha_{m}}^{\prime}= \\
=2 \alpha_{m}^{2}\left[-\frac{1}{\alpha_{m}^{3}} J_{\frac{2}{3}}\left(\frac{2}{3} \alpha_{m}^{3}\right)+J_{-\frac{1}{3}}\left(\frac{2}{3} \alpha_{m}^{3}\right)+\frac{1}{\alpha_{m}^{2}} J_{-\frac{2}{3}}\left(\frac{2}{3} \alpha_{m}^{3}\right)+J_{-\frac{1}{3}}\left(\frac{2}{3} \alpha_{m}^{3}\right)\right]= \\
=2 \alpha_{m}^{2}\left(J_{\frac{1}{3}}\left(\frac{2}{3} \alpha_{m}^{3}\right)+J_{-\frac{1}{3}}\left(\frac{2}{3} \alpha_{m}^{3}\right)\right)
\end{gathered}
$$

we have

$$
\begin{aligned}
\underset{z=\alpha_{m}}{\operatorname{res}} g(z) & =\lim _{z \rightarrow \alpha_{m}}\left[\left(z-\alpha_{m}\right)^{2} \frac{G(f(z, x))}{\alpha_{m} u^{\prime}\left(\alpha_{m}\right)^{2}\left(z-\alpha_{m}\right)^{2}+c m\left(z-\alpha_{m}\right)^{4}+\cdots}\right]^{\prime}= \\
& =\lim _{z \rightarrow \alpha_{m}} \frac{G_{z}^{\prime}(f(z, x))}{\alpha_{m} u^{\prime}\left(\alpha_{m}\right)^{2}}=\frac{2 \alpha_{m} \psi\left(\alpha_{m}^{2} x\right)^{2}}{4 \alpha_{m}^{5}\left(J_{\frac{1}{3}}\left(\frac{2}{3} \alpha_{m}^{3}\right)+J_{-\frac{1}{3}}\left(\frac{2}{3} \alpha_{m}^{3}\right)\right)} .
\end{aligned}
$$

Take as a contour of integration a rectangular contour $C$ with vertices at the points $\pm A_{N}, \pm A_{N}++i B$, which bypasses points $\alpha_{m}$ above real axis, $-\alpha_{m}$ below it.

Consider the right hand side of the contour with vertices at $A_{N}$ and $A_{N}+i B$. By using the asymptotics

$$
\begin{gathered}
J_{\frac{1}{3}}(z)+J_{-\frac{1}{3}}(z) \sim e^{-i z}, \\
J_{\frac{2}{3}}(z)-J_{-\frac{2}{3}}(z)=\frac{\left(J_{\frac{1}{3}}(z)+J_{-\frac{1}{3}}(z)\right)^{\prime}}{-2 z^{2}}+J_{\frac{1}{3}}(z)+J_{-\frac{1}{3}}(z) \sim \frac{-i e^{-i z}}{2 z^{2}}+e^{-i z}, \\
\sqrt{z^{2}-x} \sim z^{3}-\frac{3}{2} x z .
\end{gathered}
$$


For $x>0, N \rightarrow \infty$ taking $B=A_{N}, z=u+i v$ we have

$$
\begin{aligned}
& \int_{0}^{\infty} q_{k}(x) \int_{0}^{A_{N}} A_{N}^{3} \frac{e^{3 A_{N}^{2} v-v^{3}-\frac{3}{2} x v}}{e^{3 A_{N}^{2} v-v^{3}}} d v d x= \\
& =\int_{0}^{\infty} q_{k}(x)\left[A_{N}^{3} \frac{e^{-\frac{3}{2} x A_{N}}}{-\frac{3}{2} x}+\frac{2 A_{N}^{3}}{3 x}\right] d x .
\end{aligned}
$$

From condition 2

$$
\int_{0}^{\infty} \frac{q_{k}(x)}{x} A_{N}^{3} d x=0
$$

By conditions $2-3$ as $N \rightarrow \infty$

$$
\begin{gathered}
\int_{0}^{\infty}\left|\frac{q_{k}(x)}{x}\right| A_{N}^{3} e^{-\frac{3}{2} x A_{N}} d x= \\
=\int_{0}^{\infty}\left|\frac{q_{k}(x)}{x}\right| \frac{A_{N}^{3}}{1+\frac{3}{2} x A_{N}+\frac{\left(\frac{3}{2} x A_{N}\right)^{2}}{2 !}+\frac{\left(\frac{3}{2} x A_{N}\right)^{3}}{3 !}+\frac{\left(\frac{3}{2} x A_{N}\right)^{4}}{4 !}+\cdots} d x< \\
<\int_{0}^{\infty}\left|\frac{q_{k}(x)}{x}\right| \frac{1}{\frac{\left(\frac{3}{2} x A_{N}\right)^{4}}{4 !}} d x=\frac{\text { const }}{A_{N}} \int_{0}^{\infty}\left|\frac{q_{k}(x)}{x^{5}}\right| d x \rightarrow 0 .
\end{gathered}
$$

On the side of the contour with the vertices at $\pm A_{N}+i B$

$$
\begin{gathered}
\int_{0}^{\infty} q_{k}(x) \int_{-A_{N}+i B}^{A_{N}+i B} g(z) d z d x . \sim \int_{0}^{\infty} q_{k}(x) \int_{-A_{N}}^{A_{N}} e^{-\frac{3}{2} x A_{N}} A_{N}^{3} d u d x= \\
\quad=\int_{0}^{\infty} 2 q_{k}(x) A_{N}^{4} e^{-\frac{3}{2} x A_{N}} d x<\frac{\text { const }}{A_{N}} \int_{0}^{\infty} \frac{q_{k}(x)}{x^{5}} d x \rightarrow \infty .
\end{gathered}
$$

In the same way as it is done in (2.25), (2.26) we get that

$$
\lim _{N \rightarrow \infty} \int_{0}^{\infty} q_{k}(x) \int_{-A_{N}+i B}^{A_{N}+i B} g(z) d z d x=0
$$

Similarly, one may show that the integral along the left hand side of the contour converges to zero:

$$
\lim _{N \rightarrow \infty} \int_{0}^{\infty} q_{k}(x) \int_{C} g(z) d z d x=0 .
$$

So, by the Cauchy theorem we finally get

$$
\sum_{m=1}^{\infty} \int_{0}^{\infty} \frac{\psi\left(\alpha_{m}^{2}, x\right)^{2} q_{k}(x) d x}{\alpha_{m}^{4}\left(J_{\frac{1}{3}}\left(\frac{2}{3} \alpha_{m}^{3}\right)+J_{\frac{1}{3}}\left(\frac{2}{3} \alpha_{m}^{3}\right)\right)^{2}}=\lim _{N \rightarrow \infty} \int_{0}^{\infty} q_{k}(x) \int_{C} g(z) d z d x=0
$$


which completes the proof of the theorem.

\section{Acknowledgements}

This study was supported by the Science Development Foundation under the President of the Republic of AzerbaijanGrant No EIF-2011-1(3)-82/18-1.

\section{Author details}

${ }^{1}$ Institute of Mathematics and Mechanics of NAS of Azerbaijan, Baku, Azerbaijan ${ }^{2}$ Mathematics Department, Khazar

University, Baku, Azerbaijan

\section{Competing interests}

The author declares that they have no competing interests.

Received: 14 September 2011 Accepted: 23 January 2012 Published: 23 January 2012

\section{References}

1. Pechentsov, AS: Traces of one class singular differential operators: method of Lidskii-Sadovnichii. Vestnik Moscow Univ Ser I Math Mech. 5, 35-42 (1999)

2. Gasymov, MG, Levitan, BM: About sum of differences of two singular Sturm-Liouville operators. Dokl AN SSSR. 151(5):1014-1017 (1953)

3. Rybak, MA: About asymptotic of eigenvalues of some boundary value problems for operator Sturm-Liouville equation. Ukr Math J. 32(2):248-252 (1980)

4. Qorbachuk, VI, Rybak, MA: About self-adjoint extensions of minimal operator generated by Sturm-Liouville expression with operator potentials and nonhomogeneous bondary conditions. Dokl AN URSR Ser A. 4, 300-304 (1975)

5. Aliyev, BA: Asymptotic behavior of eigenvalues of one boundary value problem for elliptic differential operator equation of second order. Ukr Math J. 5(8):1146-1152 (2006)

6. Bayramoglu, M, Aslanova, NM: Distribution of eigenvalues and trace formula of operator Sturm-Liouville equation. Ukr Math J. 62(7):867-877 (2010)

7. Aslanova, NM: Study of the asymptotic eigenvalue distribution and trace formula of second order operator-differential equation. J Bound Value Probl. 7, 13 (2011)

8. Maksudov, FG, Bayramoglu, M, Adygozalov, AA: On regularized trace of operator Sturm-Liouville on finite segment with unbounded operator coefficient. Dokl AN SSSR. 277(4):795-799 (1984)

9. Bayramoglu, M, Sahinturk, H: Higher order regularized trace formula for the regular Sturm-Liouville equation contained spectral parameter in the boundary condition. Appl Math Comput. 186(2):1591-1599 (2007). doi:10.1016/j. amc.2006.08.066

10. Aslanova, NM: Trace formula of one boundary value problem for Sturm-Liouville operator equation. Sib J Math. 49(6):1207-1215 (2008)

11. Sadovnichii, VA, Podolskii, VE: Traces of operators. Uspekh Math Nauk. 61:5(371):89-156 (2006)

12. Gradstein, IS, Ryzhik, IM: Table of Integrals, Sums, Series and Products, p. 1108. Nauka, Moscow (1971)

13. Qorbachuk, VI, Qorbachuk, ML: On some classes of boundaryvalue problems for Sturm-Liouville equation with operatorvalued potential. Ukr Math J. 24(3):291-305 (1972)

doi:10.1186/1687-2770-2012-8

Cite this article as: Aslanova: The asymptpotics of eigenvalues and trace formula of operator associated with one singular problem. Boundary Value Problems 2012 2012:8.

\section{Submit your manuscript to a SpringerOpen ${ }^{\odot}$ journal and benefit from:}

- Convenient online submission

Rigorous peer review

- Immediate publication on acceptance

- Open access: articles freely available online

- High visibility within the field

- Retaining the copyright to your article

Submit your next manuscript at $\boldsymbol{s p r i n g e r o p e n . c o m ~}$ 